\title{
Molecular classification of a complex structural rearrangement of the $R B 1$ locus in an infant with sporadic, isolated, intracranial, sellar region retinoblastoma
}

Kathleen M. Schieffer ${ }^{{ }^{*}+}$ (D), Alexander Z. Feldman ${ }^{2 \dagger}$, Esko A. Kautto $^{1}$, Sean McGrath ${ }^{1}$, Anthony R. Miller ${ }^{1}$, Maria Elena Hernandez-Gonzalez ${ }^{1}$, Stephanie LaHaye ${ }^{1}$, Katherine E. Miller ${ }^{1}$, Daniel C. Koboldt ${ }^{1,3}$, Patrick Brennan ${ }^{1}$, Benjamin Kelly ${ }^{1}$, Amy Wetzel ${ }^{1}$, Vibhuti Agarwal ${ }^{4}$, Margaret Shatara ${ }^{5}$, Suzanne Conley ${ }^{6}$, Diana P. Rodriguez ${ }^{7}$, Rolla Abu-Arja ${ }^{6}$, Ala Shaikhkhalil ${ }^{8}$, Matija Snuderl ${ }^{9}$, Brent A. Orr ${ }^{10}$, Jonathan L. Finlay ${ }^{6,11,12}$, Diana S. Osorio 3,6,11, Annie I. Drapeau 13,14, Jeffrey R. Leonard ${ }^{13,14}$, Christopher R. Pierson 15,16,17, Peter White ${ }^{1,3}$, Vincent Magrini $^{1,3}$, Elaine R. Mardis ${ }^{1,3,14}$, Richard K. Wilson ${ }^{1,3}$, Catherine E. Cottrell ${ }^{1,3,16}$ and Daniel R. Boué ${ }^{15,16}$

\begin{abstract}
Retinoblastoma is a childhood cancer of the retina involving germline or somatic alterations of the RB Transcriptional Corepressor 1 gene, RB1. Rare cases of sellar-suprasellar region retinoblastoma without evidence of ocular or pineal tumors have been described. A nine-month-old male presented with a sellar-suprasellar region mass. Histopathology showed an embryonal tumor with focal Flexner-Wintersteiner-like rosettes and loss of retinoblastoma protein (RB1) expression by immunohistochemistry. DNA array-based methylation profiling confidently classified the tumor as pineoblastoma group A/intracranial retinoblastoma. The patient was subsequently enrolled on an institutional translational cancer research protocol and underwent comprehensive molecular profiling, including paired tumor/ normal exome and genome sequencing and RNA-sequencing of the tumor. Additionally, Pacific Biosciences (PacBio) Single Molecule Real Time (SMRT) sequencing was performed from comparator normal and disease-involved tissue to resolve complex structural variations. RNA-sequencing revealed multiple fusions clustered within 13q14.1-q21.3, including a novel in-frame fusion of RB1-SIAH3 predicted to prematurely truncate the RB1 protein. SMRT sequencing revealed a complex structural rearrangement spanning 13q14.11-q31.3, including two somatic structural variants within intron 17 of RB1. These events corresponded to the RB1-SIAH3 fusion and a novel RB1 rearrangement expected to correlate with the complete absence of RB1 protein expression. Comprehensive molecular analysis, including DNA array-based methylation profiling and sequencing-based methodologies, were critical for classification and understanding the complex mechanism of RB1 inactivation in this diagnostically challenging tumor.
\end{abstract}

\footnotetext{
*Correspondence: Kathleen.Schieffer@nationwidechildrens.org

${ }^{\dagger}$ KathleenM. Schieffer and AlexanderZ. Feldman have contributed equally

to this work

${ }^{1}$ The Steve and Cindy Rasmussen Institute for Genomic Medicine,

Abigail Wexner Research Institute At Nationwide Children's Hospital, 575

Children's Crossroad, Columbus, OH 43215, USA

Full list of author information is available at the end of the article
}

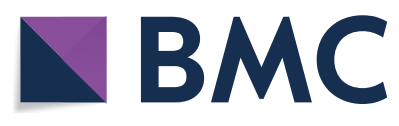

(c) The Author(s) 2021. Open Access This article is licensed under a Creative Commons Attribution 4.0 International License, which permits use, sharing, adaptation, distribution and reproduction in any medium or format, as long as you give appropriate credit to the original author(s) and the source, provide a link to the Creative Commons licence, and indicate if changes were made. The images or other third party material in this article are included in the article's Creative Commons licence, unless indicated otherwise in a credit line to the material. If material is not included in the article's Creative Commons licence and your intended use is not permitted by statutory regulation or exceeds the permitted use, you will need to obtain permission directly from the copyright holder. To view a copy of this licence, visit http://creativecommons.org/licenses/by/4.0/. The Creative Commons Public Domain Dedication waiver (http://creativeco mmons.org/publicdomain/zero/1.0/) applies to the data made available in this article, unless otherwise stated in a credit line to the data. 
Keywords: Intracranial retinoblastoma, Sellar-suprasellar retinoblastoma, RB1, Structural variation, DNA array-based methylation, SMRT sequencing, PacBio

\section{Introduction}

Retinoblastoma is a childhood cancer predominately occurring intraocularly, with an estimated prevalence of 1 per 16,000-18,000 live births and approximately 8,000 new cases per year [3]. It is characterized by biallelic inactivation of the RB Transcriptional Corepressor 1 gene ( $R B 1)$ which encodes the retinoblastoma protein (RB1), a well-established tumor suppressor that interacts with the E2F family of transcription factors to negatively regulate the cell cycle. As described by Knudson's "twohit hypothesis," individuals with hereditary retinoblastoma present with a germline heterozygous alteration in $R B 1$. Somatic inactivation of the second $R B 1$ allele results in the development of retinoblastoma [14]. Approximately $40 \%$ of individuals with retinoblastoma have a hereditary form, commonly associated with bilateral retinoblastoma (i.e., dual primary tumors presenting in both eyes) early in life [21, 22]. Unilateral retinoblastoma most frequently arises in individuals with biallelic somatic alterations in the developing retina [3]. Rarely, individuals may develop bilateral retinoblastoma together with synchronous or asynchronous intracranial pineal and/or sellar region tumors, i.e. involving the sella turcica/pituitary gland area (so-called trilateral and/or "quadrilateral" retinoblastoma) [6]. Intracranial central nervous system (CNS) tumors are identified prior to ocular retinoblastoma diagnosis in only $3 \%$ of cases $[5,35]$.

Intracranial sellar-suprasellar region retinoblastoma without evidence of ocular or pineal tumor is an exceedingly rare occurrence, with only a single report described in the literature to our knowledge [13]. An early case series reported an infant with isolated suprasellar tumor, without retinal involvement [13]. This patient was noted to have a positive family history, including bilateral retinoblastoma in a sibling and paternal unilateral retinoblastoma. A second report described an infant with biallelic somatic $R B 1$ inactivating alterations (LRG_517t1:c.T494A;p.Leu165", LRG_517t1:c.717dup;p. Lys $\left.249^{*}\right)$ in the absence of a germline $R B 1$ alteration, who presented with an ectopic, intracranial, sellar-suprasellar region retinoblastoma, but also had a smaller similarappearing pineal mass detected on imaging [23]. In the latter case, the diagnosis was supported by DNA arraybased methylation profiling on the sellar region tumor; the pineal region tumor was not sampled. Additional data are necessary to better understand the frequency and genomic mechanisms associated with isolated, ectopic, intracranial, sellar-suprasellar region retinoblastoma.
The genomic landscape of retinoblastoma is diverse and may result from a spectrum of events, including single nucleotide variations (SNVs), small insertion-deletion (indel) events, splice site alterations, copy number alterations (CNAs), loss of heterozygosity (LOH), and promoter hypermethylation $[15,21,22]$. Highly penetrant germline alterations consist of predominately loss-offunction variants (e.g. nonsense, frameshift, canonical splice site) predicted to encode a premature stop of translation in the protein [21]. Low penetrance alterations are thought to reduce gene expression or partially inactivate the RB1 protein and may be associated with promoter variants, non-canonical splice site alterations, missense variants, and small non-frameshift deletions [8, 32]. In an estimated $60-70 \%$ of hereditary and non-heritable tumors, the initial $R B 1$ alteration is followed by $\mathrm{LOH}$ at the 13q14.2 locus, encompassing $R B 1$ [21]. Chromothripsis involving 13q14.2 has been described as an exceedingly rare mechanism of $R B 1$ inactivation in individuals with somatic disease $[15,20]$. In three retinoblastoma patients, chromothripsis disrupted the $R B 1$ locus resulting in a gene fusion, and immunohistochemical (IHC) staining demonstrated complete absence of RB1 protein expression in the tumor [20]. Additionally, genome sequencing of retinoblastoma tumors without a previously identified $R B 1$ alteration revealed large structural rearrangements encompassing the $R B 1$ locus with variable complexity [2]. Beyond the $R B 1$ gene, amplification of the proto-oncogene $M Y C N$ has been reported in a small subset $(\sim 2 \%)$ of sporadic retinoblastoma cases without RB1 alterations [24].

Herein, we describe a patient with a sporadic, isolated, intracranial, sellar-suprasellar region retinoblastoma with diagnostic refinement by DNA array-based methylation profiling as well as comprehensive genomic profiling of germline and somatic tissues. Complex structural rearrangement of 13q14.11-q31.3 was identified, including two somatic $R B 1$ gene rearrangements. Considerations for therapeutic management are presented.

\section{Case Presentation}

\section{Clinical history and diagnostic work-up}

A nine-month-old male with no significant past medical history initially presented with two months of worsening fussiness and two days of vomiting. His physical exam was unremarkable and there was no history of polyuria or polydipsia. A magnetic resonance imaging (MRI) of the brain revealed a $3.2 \times 3.0 \times 2.6 \mathrm{~cm}$ lobular 
sella-suprasellar mass, with avid contrast enhancement (Fig. 1a-c). The mass was separate from the optic chiasm, eroded the dorsum sella, and extended into the subfrontal region. Intralesional hemorrhage was noted as well (not shown). An MRI of the spine was negative for metastases. The primary radiologic differential was germ cell tumor vs. atypical teratoid/rhabdoid tumor. An endocrinology workup confirmed central adrenal insufficiency requiring maintenance and stress doses of hydrocortisone. A craniotomy was performed, and the tumor was found to be adherent to surrounding critical structures. A subtotal resection was achieved.

Hematoxylin and eosin (H\&E) stained sections demonstrated a small cell embryonal neoplasm characterized by sheets of poorly differentiated cells with nuclear pleomorphism, nuclear molding, and occasional apoptotic bodies and mitoses (Fig. 2a). Rare rosettes were appreciated, composed of a single layer of tumor cells with short cytoplasmic processes extending into an otherwise empty lumen (Flexner-Wintersteiner-like rosettes, Fig. 2b).
Rhabdoid inclusions, abundant neuropil, perivascular rosettes, microvascular proliferation, necrosis, primitive neural tubes, and/or glandular structures were not seen.

Synaptophysin IHC demonstrated diffuse and strong positivity (Fig. 2c). The Ki-67 labeling index was approximately $40-50 \%$ (Fig. 2d). By IHC, tumor cells showed only focal, weak staining or were negative for Olig2, BCL-6 interacting corepressor protein (BCOR), nuclear protein in testis family member 1 protein (NUTM1), neuronal nuclei protein (Neu-N), neurofilament, glial fibrillary acid protein (GFAP), p53, SALL4, epithelial membrane antigen (EMA), and lin-28 homolog A (Lin28A). Tumor cells retained both integrase interactor 1 (INI-1) and transcription activator BRG1 (BRG1). Fluorescence in-situ hybridization (FISH) was negative for rearrangements of $B C O R$, capicua transcriptional repressor gene $(C I C)$, or Ewing sarcoma breakpoint region 1 (EWSR1) and negative for amplification of the C19MC locus at 19q13.42. DNA array-based methylation profiling confidently classified the tumor as pineoblastoma

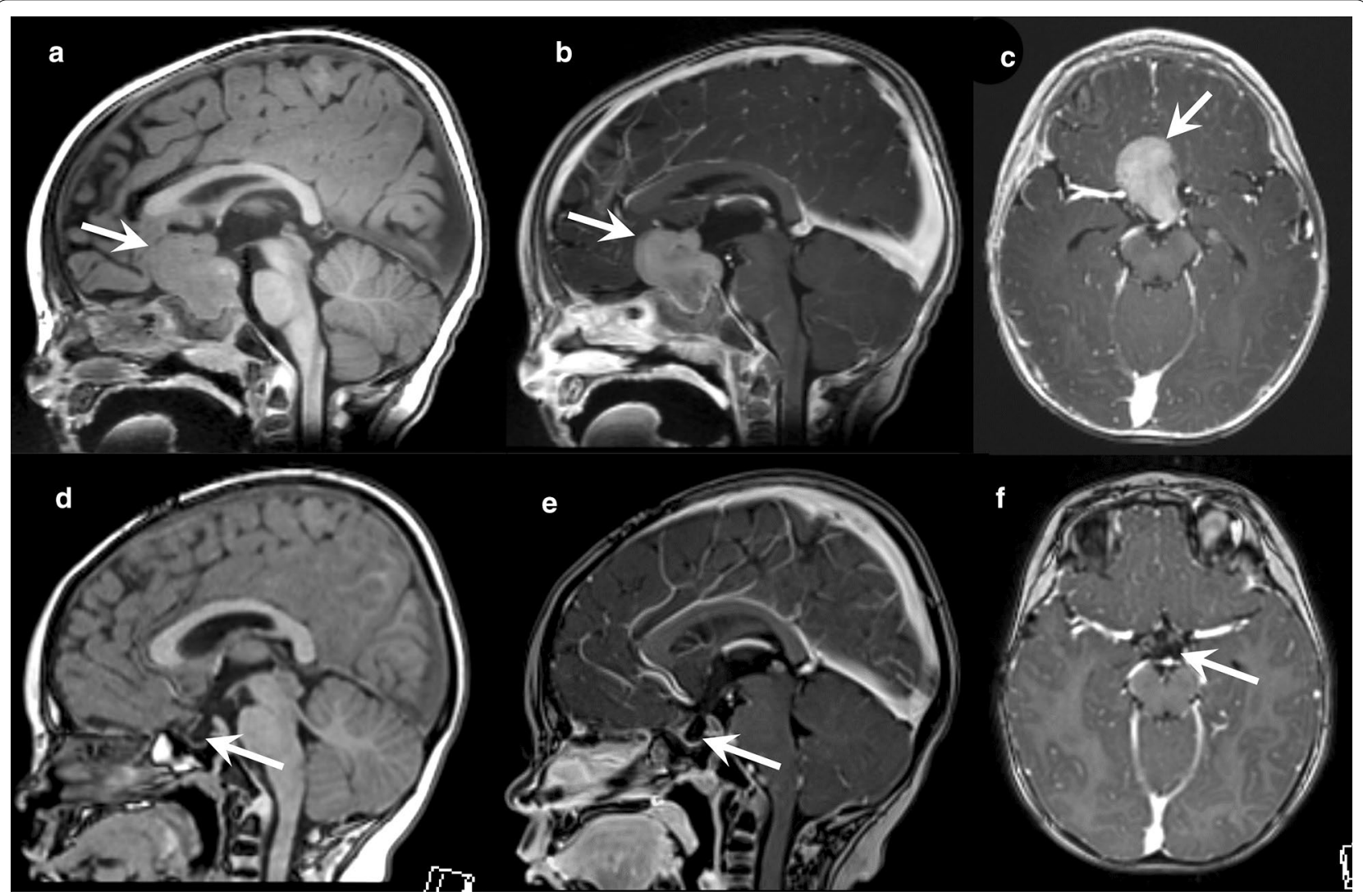

Fig. 1 At diagnosis: Pre contrast sagittal T1-W image (a), post contrast sagittal T1-W image (b), and post contrast axial T1-W image (c) show a large lobulated avidly enhancing mass that fills and expands the sella, and extends to the suprasellar cistern (arrows). This mass is separated from the optic nerves and optic chiasm, and contacts the circle of Willis vessels, which preserve normal caliber. At completion of therapy: Pre contrast sagittal T1-W image (d), post contrast sagittal T1-W image (e), and post contrast axial T1-W image (f) show complete resolution of the sellar, suprasellar mass with no evidence of residual tumor, an empty sella (arrows), and normal surrounding structures 


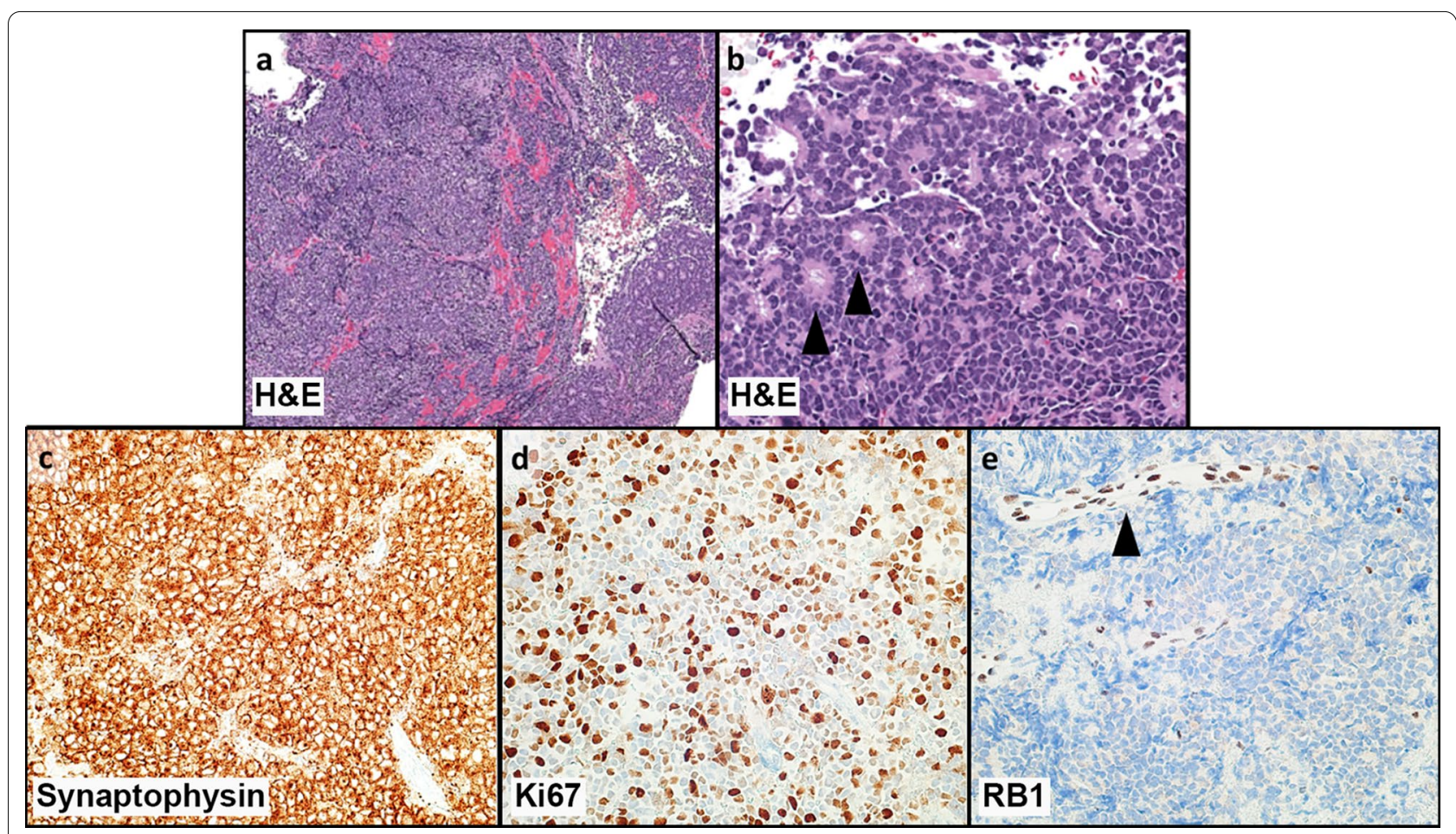

Fig. 2 Tumor histology and immunohistochemistry. a Hematoxylin and eosin (H\&E) sections reveal an embryonal neoplasm composed of mostly patternless sheets of small tumor cells, $40 \times$. b At higher magnification, the tumor cells are poorly differentiated, with nuclear pleomorphism, some nuclear molding, and occasional apoptotic bodies and mitoses. Rare Flexner-Wintersteiner-like rosettes were seen focally (arrowheads) 200x. c Synaptophysin immunohistochemistry (IHC) is diffusely, strongly positive, 200x. d Ki-67 tumor nuclear labeling index is approximately 40-50\%, $200 \times$. (e) IHC for RB Transcriptional Corepressor 1 (RB1) protein demonstrates loss of constitutive nuclear staining in tumor cell nuclei, but retained normal staining in vascular endothelial cell nuclei (arrowhead), 200x

group A/intracranial retinoblastoma (calibrated score: 0.99) [1]. Subsequently, IHC staining for RB1 protein was performed, and showed complete loss of normal constitutive nuclear expression in tumor cells, with retained expression in tumor vascular endothelial nuclei (Fig. 2e).

The patient was enrolled on the Head Start 4 clinical trial (NCT02875314) with the diagnosis of CNS embryonal tumor, not otherwise specified (NOS) and underwent five induction cycles of intensive chemotherapy with $12 \mathrm{~g} / \mathrm{m}^{2}(400 \mathrm{mg} / \mathrm{kg})$ of methotrexate. Radiologic evaluation at the completion of therapy demonstrated no evidence of residual tumor (Fig. 1d-f). He was randomized into tandem autologous hematopoietic stem-cell rescues with carboplatin and thiotepa. His post-operative and overall clinical course were complicated by partial pituitary dysfunction (hypothyroidism, adrenal insufficiency), bacterial and fungal sepsis, pneumatosis coli and feeding challenges, and compression fracture. However, the patient remained without significant CNS deficits.

$\mathrm{He}$ underwent periodic sedated ophthalmologic evaluations to visualize the retinae during scheduled surveillance MRIs, which showed no evidence of retinal disease. Retinal surveillance was discontinued at
2 years of age as development of classic retinoblastoma would be unlikely after this age. He is currently 3 years old and 1.5 years off-therapy and doing well with continued follow-up by gastroenterology for his feeding challenges. He tolerates full enteral calories via G-tube, has started making steady progress in improving his oral intake, and is gaining weight well.

\section{Materials and methods}

The patient was enrolled as part of an Institutional Review Board (IRB) approved study at The Steve and Cindy Rasmussen Institute for Genomic Medicine (IGM) at Nationwide Children's Hospital (NCH). Informed consent was provided by the patient's parents for comprehensive genomic analysis. Peripheral blood mononuclear cells (PBMCs) were collected by routine venipuncture for genomic DNA extraction. Snap-frozen tumor tissue was obtained for DNA and RNA extraction (estimated $80 \%$ tumor cellularity). Full materials and methods can be found in the Supplementary Information (Additional File 1: Materials and Methods). 


\section{Molecular characterization of intracranial retinoblastoma reveals RB1 loss-of-function due to gene fusion}

Comprehensive molecular profiling, including paired tumor/normal genome sequencing (GS) and enhanced exome sequencing (eES) were performed using Illumina next-generation sequencing (NGS) to evaluate germline variations, somatic SNVs, small indels, structural variations (SVs), and CNAs. Notably, we did not identify any pathogenic germline alterations in well-established cancer predisposition genes [36], with the RB1 and DICER1 loci assessed at moderate depth within the GS germline data $(>70 \times$ and $>100 \times$ coverage, respectively) and at high depth in eES germline data $(>400 \times$ and $\sim 450 \times$ coverage, respectively). Although the tumor did not harbor any clearly medically meaningful somatic SNVs or small indels, we identified a gain on chromosomal arm 1q and a focal deletion on $13 \mathrm{q}$ distal to, but not encompassing, RB1 (Additional File 1: Fig. S1a-b). This focal deletion (hg19: chr13:57,297,261-58,439,492) does not contain any genes associated with cancer. We did not identify any CNAs or LOH associated with the RB1 locus. RNAsequencing of the tumor tissue revealed two gene fusion events, clustered within chromosomal bands 13q14.1q21.3, RB1-SIAH3 and ZC3H13-KLHL1, and two interchromosomal fusions between chromosomes 10 and 13 (DLEU1-DNAJC12 and RBP3-TPT1) (Table 1). ZC3H13KLHL1, DLEU1-DNAJ12, and RBP3-TPT1 are not recurrent fusions in cancer nor are the genes involved in these events recurrently altered in pediatric cancers. Therefore, the association of these events with tumorigenesis is unknown. We performed reverse transcriptase polymerase chain reaction (RT-PCR) followed by Sanger sequencing to confirm the RB1-SIAH3 fusion and verify the inferred translational protein frame. The in-frame fusion event involved exon 17 of RB1 (NM_000321) and exon 2 of SIAH3 (NM_198849), resulting in a 789 amino acid chimeric protein which is predicted to prematurely truncate $R B 1$ approximately $60 \%$ of the way through the translated protein (Fig. 3a). The fusion retains pocket domain $\mathrm{A}$ of the RB1 protein but demonstrates loss of both the linker domain and pocket domain B (Fig. 3b). Interaction of pocket domains $\mathrm{A}$ and $\mathrm{B}$ with the linker domain is important for supporting the tumor suppressor role of RB1 [9]. Read coverage of this $R B 1$ transcript (NM_000321), as derived from RNA sequencing, revealed minimal reads supporting full-length $R B 1$, with a loss of sequence read depth at the fusion breakpoint (exon 17) through the $3^{\prime}$ terminus (Fig. 3c). The loss of full-length $R B 1$ expression is consistent with the absence of $\mathrm{RB1}$ protein demonstrated in the tumor by IHC (Fig. 2e). Given an estimated tumor content of $80 \%$ in the sequenced tumor tissue, the low number of detected full-length transcript reads likely correspond to admixed normal brain and connective tissue, as demonstrated by retained RB1 expression in tumor vascular endothelial nuclei by IHC (Fig. 2e).

\section{Complex chromosome $13 q$ structural rearrangement identified through SMRT sequencing methodologies}

Given the identification of multiple gene fusions near the $R B 1$ gene locus and the complete loss of $\mathrm{RB} 1$ protein expression by IHC, we utilized PacBio Single Molecule Real Time (SMRT) sequencing-based methodologies to resolve the genomic event. Evaluation of RNA by SMRT sequencing used the Iso-Seq method, providing reads that span entire transcripts to enable the characterization of full-length transcript isoforms. Iso-Seq sequencing of full-length transcript isoforms was utilized to elucidate the diversity of fusions in proximity to and encompassing the RB1 locus. We confirmed the presence of both RB1-SIAH3 (Fig. 4a, Additional File 1: Fig. S2) and ZC3H13-KLHL1 in-frame fusion events (Additional File 1: Fig. S3a) by observing Iso-Seq reads with split alignments between the fusion partners. The ZC3H13-KLHL1 chimeric protein is predicted to retain domains important for protein function, including the Broad-complex, Tramtrack, Bric-a-Brac/Poxvirus Zinc finger (BTB/POZ) domain and Kelch repeats (Additional File 1: Fig. S3b); however, the association of this fusion with tumorigenesis is uncertain [7]. The RB1-SIAH3 fusion was noted

Table 1 Gene fusions surrounding the $R B 1$ locus at 13q14.1-q21.3

\begin{tabular}{|c|c|c|c|c|c|c|}
\hline Fusion & $5^{\prime}$ gene partner & $\begin{array}{l}5^{\prime} \text { gene partner } \\
\text { coordinates (GRCh38), } \\
\text { exon }\end{array}$ & Cytoband & $3^{\prime}$ gene partner & $\begin{array}{l}\text { 3' gene partner } \\
\text { coordinates (GRCh38), } \\
\text { exon }\end{array}$ & Cytoband \\
\hline RB1-SIAH3 & RB1 (NM_000321) & chr13:48,381,443, exon 17 & $13 q 14.2$ & SIAH3 (NM_198849) & chr13:45,784,057, exon 2 & $13 q 14.13$ \\
\hline ZC3H13-KLHL1 & ZC3H13 (NM_001076788) & chr13:46,003,139, exon 8 & $13 q 14.13$ & KLHL 1 (NM_020866) & chr13:69,975,802, exon 2 & $13 q 21.33$ \\
\hline DLEU1-DNAJC12 & DLEU1 (NR_109974) & chr13:50,433,550, exon 4 & $13 q 14.3$ & DNAJC12 (NM_021800) & chr10:67,823,392, exon 2 & $10 q 21.3$ \\
\hline RBP3-TPT1 & RBP3 (NM_002900) & chr10:47,349,271, exon 1 & $10 q 11.22$ & TPT1 (NM_003295) & chr13:45,341,148, exon 1 & $13 q 14.13$ \\
\hline
\end{tabular}

RB1, RB Transcriptional Corepressor 1; SIAH3, Siah E3 Ubiquitin Protein Ligase Family Member 3; ZC3H13, Zinc Finger CCCH-Type Containing 13; KLHL1, Kelch-like 1 gene; DLEU1, Deleted In Lymphocytic Leukemia 1; DNAJC12, DnaJ Heat Shock Protein Family (Hsp40) Member C12; RBP3, Retinol Binding Protein 3; TPT1, Tumor Protein, Translationally-Controlled 1 
a

$\begin{array}{ccc}\text { RB1 } & \text { fusion } & \text { SIAH3 } \\ \text { (NM_000321) } & \text { junction } & \text { (NM_198849) } \\ \text { exon 17 } & \text { V } & \text { exon 2 }\end{array}$

Ala Trp Leu Ser Tyr Val Ser Ser

GCATGGCTCTCATATGTGTCCAGT

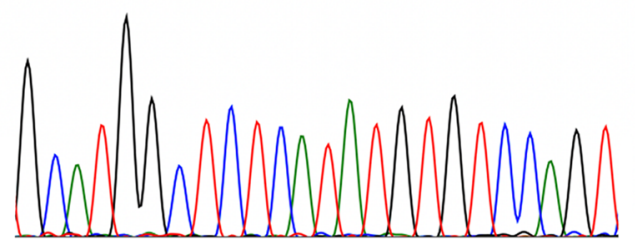

b

RB1 (NP_000312)

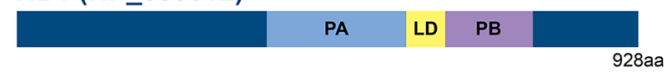

SIAH3 (NP_942146)

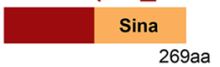

\section{RB1-SIAH3}
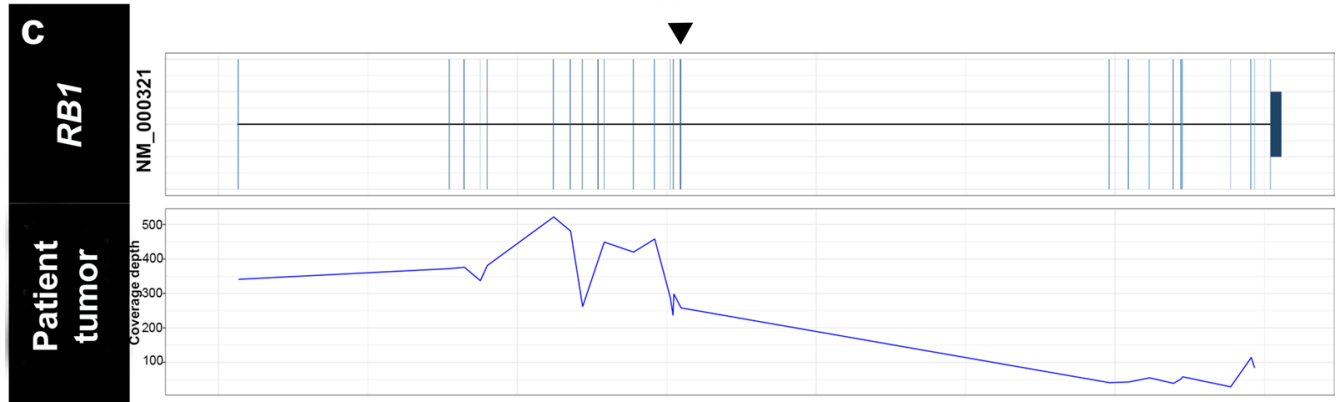

Fig. 3 Molecular characterization of an in-frame RB1-SIAH3 fusion in a pediatric intracranial retinoblastoma. a Sanger sequencing chromatogram of the in-frame RBT-SIAH3 gene fusion. The corresponding amino acid sequence is described above the chromatogram. $\mathbf{b}$ Protein domains corresponding to the RBI-SIAH3 fusion, demonstrating loss of the RB1 linker domain and pocket domain B. c RNA sequencing read coverage for RB1 (NM_000321) binned per exon. A dramatic loss of RB1 expression occurs between exons 17 (black arrowhead) and 18 at the fusion breakpoint

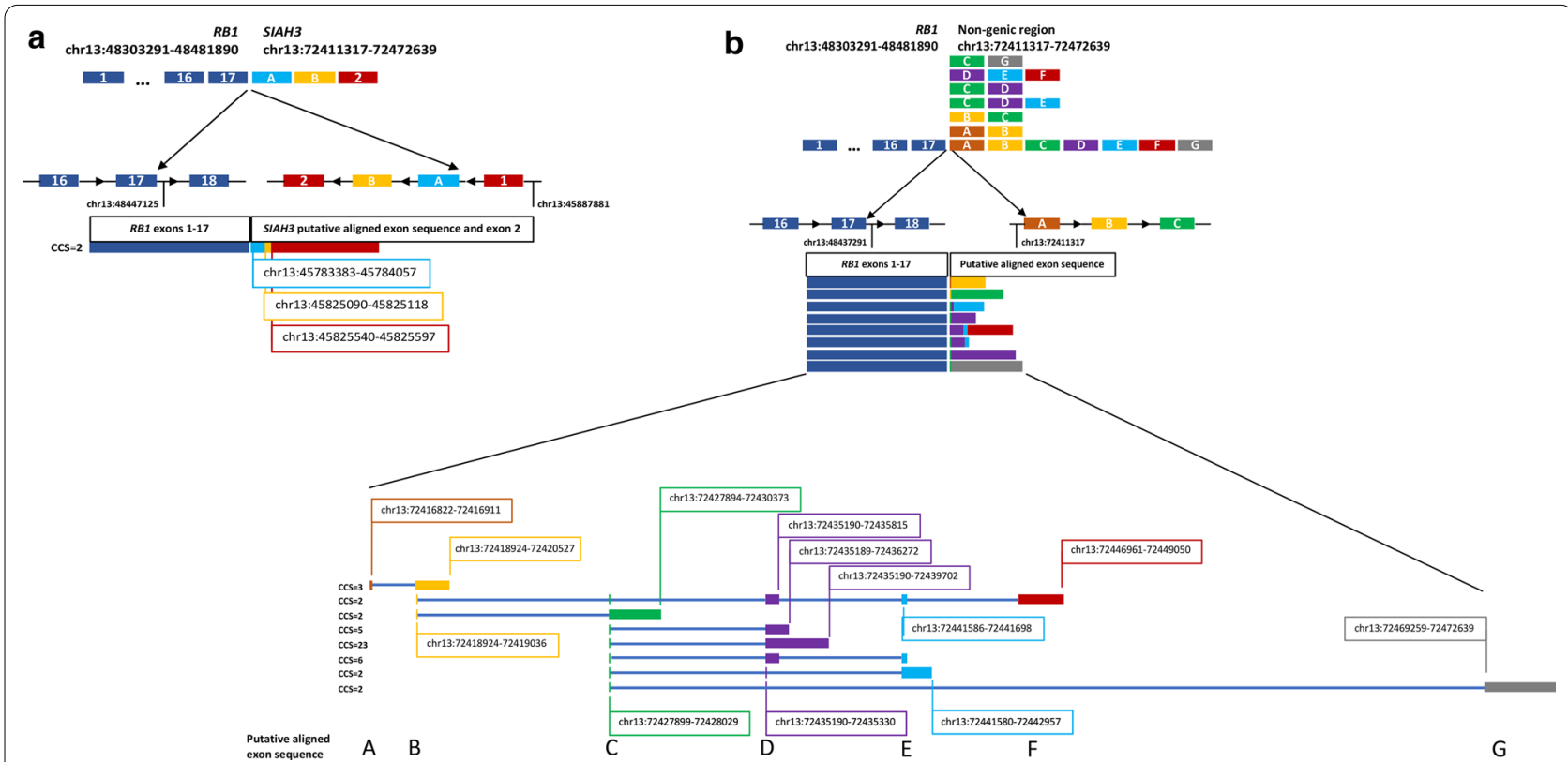

Fig. 4 PacBio SMRT sequencing by Iso-Seq resolves $R B 1$ transcript isoform structure and diversity. a SMRT sequencing of tumor-derived RNA aligned to GRCh38 identified a novel RBT-SIAH3 fusion which included putative aligned exon sequence derived from intron 1 of SIAH3 prior to the fusion junction. $\mathbf{b}$ Multiple transcript isoforms of an RB1-intergenic fusion. The intergenic sequence aligned to a non-genic region of the genome and resulted in the formation of putative aligned exon sequence supported by flanking splice donor and acceptor sequence. Each putative aligned exon is designated as A-G. The supporting number of circular consensus sequencing (CCS) reads are shown for each isoform 
to harbor putative aligned exon sequence derived from SIAH3 intervening sequence. Furthermore, we identified a novel $R B 1$-intergenic fusion aligning to a nongenic region of the genome (Fig. $4 \mathrm{~b}$ ). This fusion event presented as multiple potential isoforms, demonstrated by variability in putative aligned exon sequence supported by flanking splice donor and acceptor sequence. The predicted translation encodes a termination codon early within the putative aligned exon sequence; thus, we do not envisage a full-length chimeric protein to be produced. These findings lend support to a complex structural event that is unable to be fully resolved by NGS methodologies. To further resolve this event and predict the phase of the $R B 1$ fusion events, PacBio single-molecule HiFi circular consensus sequencing (CCS) of paired tumor/normal genomic DNA was performed
[33]. The sequencing methodology generated highly accurate ( $>99 \%$ ) reads in excess of $10 \mathrm{~kb}$. The HiFi reads were aligned to the human reference genome version 38 (GRCh38) using minimap2 and NGMLR, with structural variant calling performed with Sniffles, pbsv, and SVIM $[10,17,28]$. An ensemble-based approach was utilized to retain only SVs called by five different approaches. In the tumor tissue, predominately large inversion events spanning 13q14.11-q31.3 both involving and encompassing the $R B 1$ locus were identified (Fig. 5a, Additional File 1: Table S1).

On the basis of achieved read lengths of $>10 \mathrm{~kb}$ in $\mathrm{HiFi}$ sequencing data, we could predict the phase of certain somatic SVs in the tumor tissue. Importantly, we identified two somatic SVs hypothesized to occur in trans within intron 17 of the $R B 1$ gene based on the absence

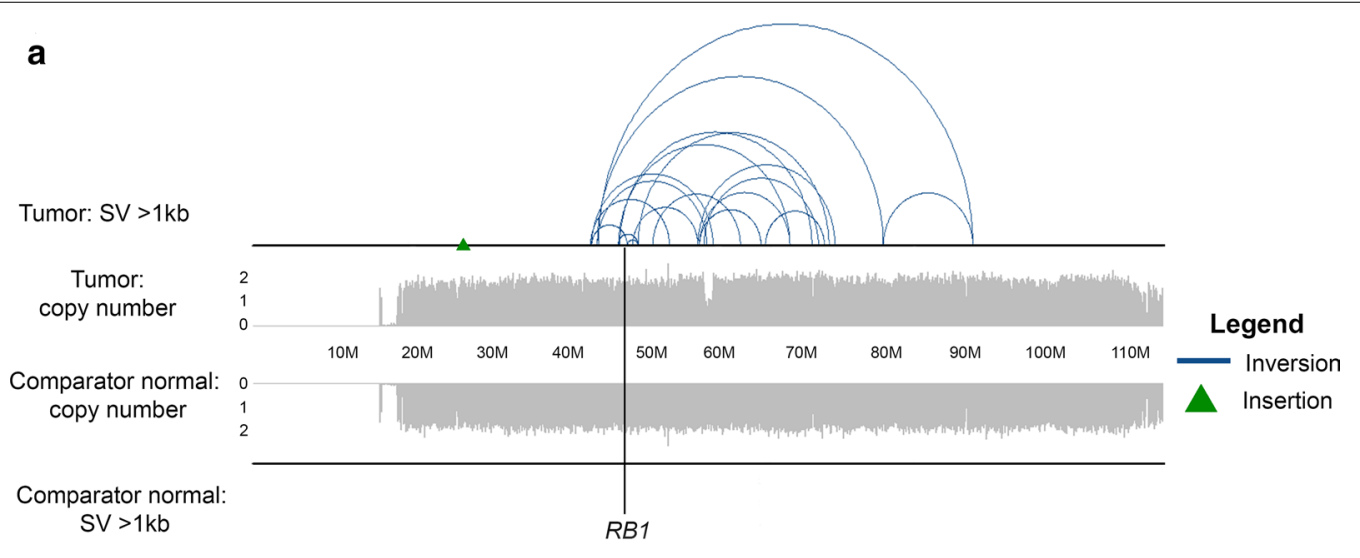

b

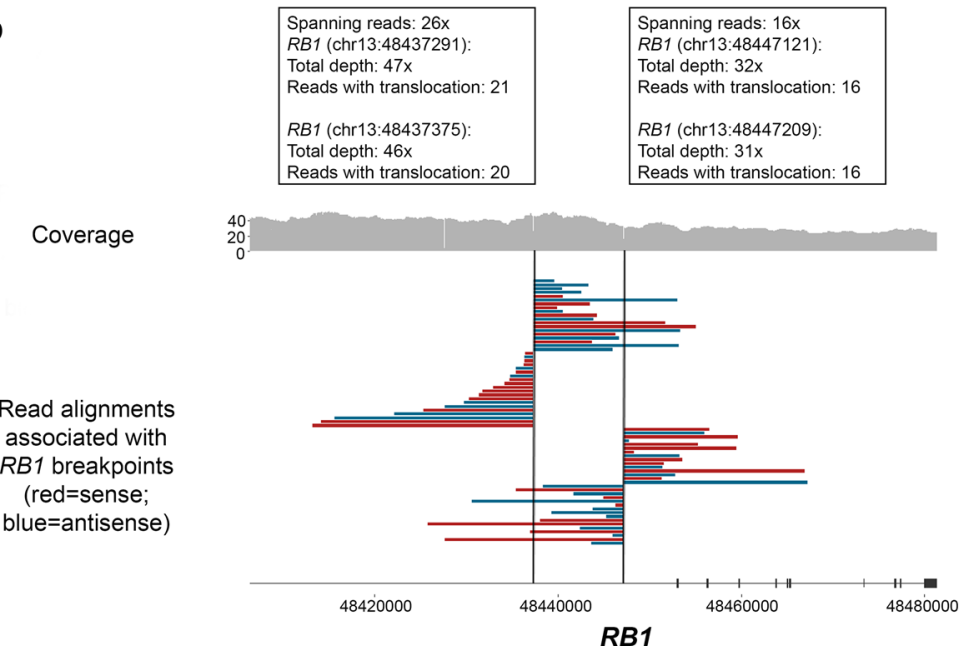

Fig. 5 PacBio SMRT sequencing of paired tumor/normal DNA resolves RB1 structural rearrangement. a Paired tumor/normal PacBio SMRT sequencing demonstrates complex structural variations (SVs) of the tumor aligned to GRCh38. Top: tumor SVs $>1$ kb and copy number median-normalized to chromosome 13 coverage. Bottom: comparator normal SVs $>1$ kb and copy number median-normalized to chromosome 13 coverage. Type of SVs denoted by a blue line (inversion) and green triangle (insertion). RB1 is denoted by the vertical black line. b Two somatic rearrangements within intron 17 of $R B 1$. Coverage calculated as average of $100 \mathrm{~kb}$ bins. Read alignments of breakpoint-associated reads in $R B 1$ are shown. Red reads are sense (+) strand, blue reads are antisense (-) strand. Lines indicate regions of coverage depth loss corresponding to RB1 SVs 
of reads spanning across both breakpoints (Fig. 5b, Additional File 1: Fig. S4). These events correspond to the $R B 1-S I A H 3$ fusion and the RB1-non-genic rearrangement previously identified by next-generation and/or SMRT sequencing methodologies, indicating the complete absence of RB1 protein expression in the tumor is associated with somatic RB1 loss-of-function SVs. Additionally, these data support the hypothesis that the driving event in this tumor is somatic in nature, rather than associated with germline $R B 1$ loss-of-function. Despite the complexity of SVs at this region, the copy number state of the chromosome is not altered; thus, it does not meet the criteria for chromothripsis [16] (Additional File 1: Fig. S5). Therefore, these events are a consequence of a larger, complex, copy-neutral structural rearrangement of chromosome 13q.

\section{Discussion and conclusions}

Herein, we described a nine-month-old male with a sporadic, isolated, intracranial, sellar-suprasellar region retinoblastoma (i.e., without evidence of preceding, concomitant, or subsequent development of retinal or pineal gland neoplasia). DNA array-based methylation classified the tumor with high confidence as pineoblastoma group A/intracranial retinoblastoma.

Given the diffuse and strong staining essentially limited to synaptophysin, following performance of an extensive IHC stain panel, many CNS and extra-CNS small cell embryonal tumors were considered in our differential diagnosis. There was no C19MC locus amplification or Lin28a IHC reactivity, making embryonal tumor with multilayered rosettes (ETMR) or related tumor unlikely. Additionally, neither IHC nor FISH detected alterations in BCOR, CIC, or NUTM1, making newly described pediatric embryonal tumors such as the CNS high-grade neuroepithelial tumor with $B C O R$ alteration and CNS Ewing sarcoma family tumor with $C I C$ alteration unlikely. The absence of EWSR1 rearrangement by FISH helped exclude intra- or extra-cranial Ewing's sarcoma. Retained nuclear expression of INI-1 and Brg-1 excluded atypical teratoid/rhabdoid tumors. Comprehensive germ cell markers, including SALL4, were negative, ruling out poorly differentiated malignant germ cell tumor. Negative staining for Olig-2 and GFAP essentially ruled out a poorly differentiated high-grade glioma/ astrocytoma. Negative staining for EMA and GFAP made a poorly differentiated ependymoma unlikely, and the negative Olig-2 made CNS neuroblastoma with FOXR2 activation unlikely. Although synaptophysin was positive, negative chromogranin staining made a poorly differentiated neuroendocrine tumor unlikely. The lack of glandular elements and lack of DICER 1 alteration made pituitary blastoma unlikely. Other negative IHC markers (e.g. myogenin, HMB-45, Neu-N, neurofilament, Oct 3/4, Glypican 3, CD1a, and Langerin) essentially excluded rhabdomyosarcoma, melanoma, malignant neuronal/ glio-neuronal neoplasms, and Langerhans cell histiocytosis. Instead, the overall findings of diffuse synaptophysin reactivity, presence of Flexner-Wintersteiner-like rosettes, DNA array-based methylation classification, detected RB1 alterations, and complete loss of RB1 expression by IHC in tumor cells, allowed a confident diagnosis of intracranial retinoblastoma.

Along with the tumor location, overall histopathology and molecular characteristics, the methylation-based classification allowed for an essentially definitive diagnosis of intracranial retinoblastoma in what would otherwise have been a difficult to classify embryonal neoplasm. Further molecular workup elucidated the mechanism of somatic $R B 1$ inactivation in this tumor in the absence of germline $R B 1$ or DICER1 alterations. As part of a clinical trial for medulloblastomas and other CNS embryonal tumors, our patient was treated with intensive chemotherapy followed by three tandem stem-cell rescues and with no evidence of residual tumor at completion of therapy. Similar regimens have been described in the treatment of other intracranial retinoblastomas, obviating the use of radiotherapy [23].

The integration of classification by genome-wide DNA array-based methylation profiling into the clinical diagnostic paradigm for CNS tumors with challenging histopathology has aided in refining both diagnosis and clinical management [12]. The utility of this methodology in combination with comprehensive genomic profiling was evident in this patient, as standard histopathological work-up originally resulted in a less-definitive diagnosis of CNS embryonal neoplasm, NOS, for this infant.

Given the differential diagnoses of intracranial retinoblastoma and pituitary blastoma, potential germline alterations of $R B 1$ and DICER1 were investigated but not found. Of clinical importance, children with hereditary $R B 1$ alterations are at increased risk for developing midline intracranial tumors, including pineal and sellar region tumors [11]. This phenomenon can be attributed to a common embryogenesis between the retinae and pineal gland, explaining the co-occurrence of retinoblastomas and pineoblastoma in patients with "trilateral" retinoblastoma. Moreover, the presence of ectopic photoreceptor cells along intracranial portions of the optic nerve system provides an explanation for the development of midline, intracranial, sellar region retinoblastoma [26, 27, 31]. Therefore, accurately identifying individuals with germline $R B 1$ alterations is critical for appropriate genetic counseling and clinical management (e.g., surveillance strategies and cancer risk assessment) [29]. Notably, diagnosis of intracranial retinoblastoma 
in an individual without hereditary retinoblastoma, incorporating DNA array-based methylation profiling, has previously been described [23], though this was in a patient with a concomitant pineal lesion. This report and the aforementioned prior study [23] highlight the utility of this methodology for this rare subset of non-heritable intracranial retinoblastoma.

Although the genomic landscape of retinoblastoma is diverse, large structural rearrangements are infrequently reported and, when described, are typically associated with chromothripsis $[2,15,21,22,30]$. Herein, the identification of the RB1-SIAH3 and ZC $3 H 13-K L H L 1$ fusions by RNA-sequencing led us to further investigate the possibility of a larger chromosomal event. RB1 and SIAH3 reside in close proximity $(\sim 2 \mathrm{Mb}$ apart $)$ to one another on chromosome 13 but are transcribed in alternate directions. The chimeric protein product of the RB1-SIAH3 fusion is predicted to truncate the linker domain and pocket domain $B$ of $R B 1$. The interaction of the RB1 pocket domains $\mathrm{A}$ and $\mathrm{B}$ with the linker domain is critical to tumor suppression and the E2F regulatory function associated with RB1 [9]. Through SMRT sequencing technologies, we identified a second $R B 1$ fusion with non-genic chromosome 13 sequence expected to disrupt RB1 protein expression. Both identified fusions demonstrated $R B 1$ breakpoints within intron 17. Although structural rearrangements in $R B 1$ are exceedingly rare, intron 17 breakpoints have been reported in multiple tumors with complex structural rearrangement in two independent studies, suggesting this may be a recurrent region for rearrangement $[2,20]$. However, larger studies are necessary to confirm this finding. These fusions are predicted to result in complete loss-of-function, as evidenced by the absence of RB1 protein expression as demonstrated by IHC herein. Through multiple methodologies, we determined that the copy number state of chromosome 13 remained neutral, despite the presence of complex structural rearrangements.

Utilization of NGS methodologies to fully elucidate the complex biological mechanism of this intracranial retinoblastoma proved challenging. However, through the use of multiple sequencing methodologies and algorithms, we identified significant overlap in the high-confidence SVs. SMRT sequencing technologies overcome many of the limitations of NGS approaches which rely on short reads, including the ability to interrogate reads $>10 \mathrm{~kb}$ in length from a single DNA molecule for SV detection [19]. These approaches are more sensitive to larger chromosomal events than NGS technologies; the reads are more likely to encompass the breakpoint or the entire structural rearrangement and can provide the advantage of predicting phased genomic variation [19, 25]. Through SMRT sequencing methodologies, we were able to confirm two somatic rearrangements within $R B 1$, predicted to be in trans, and supported by the absence of RB1 staining by IHC.

Our patient was treated on Head Start 4, a clinical trial for pediatric patients with medulloblastoma or other CNS embryonal tumors and randomized to undergo tandem autologous hematopoietic stem-cell rescues with three cycles of carboplatin and thiotepa. A prior report used a similar regimen of tandem high-dose chemotherapy with carboplatin, etoposide, cyclophosphamide, and thiotepa followed by autologous hematopoietic stem cell transplant for an infant with intracranial retinoblastoma [23]. In both our described patient and the prior case [23], a partial resection with a stable response was achieved, obviating the need for ancillary radiation therapy and its associated morbidity in this age group. Retrospective studies and case reports have shown the utilization of high-dose chemotherapy in the setting of trilateral retinoblastoma improves survival, although a standardized regimen has yet to be determined [4, 34, 35].

In summary, we report on an infant with an isolated, intracranial, sellar-suprasellar region retinoblastoma in the absence of a germline $R B 1$ alteration. A comprehensive approach to molecular profiling, including DNA array-based methylation profiling, Illumina NGS, and Pacific Bioscience SMRT sequencing, were critical for providing a definitive diagnosis and understanding the biological mechanism of $R B 1$ inactivation. The patient was treated using high-dose chemotherapy with autologous hematopoietic stem cell transplant, a regimen that has demonstrated benefit in small studies of trilateral retinoblastoma $[4,34,35]$.

\section{Abbreviations}

BCOR: $\mathrm{BCL}-6$ interacting corepressor protein; $B C O R$ : $\mathrm{BCL}-6$ interacting corepressor gene; BRG1: Transcription activator BRG1; BTB/POZ: Broad-complex, Tramtrack, Bric-a-Brac/Poxvirus Zinc finger; CCS: Circular consensus sequencing; cDNA: Complimentary deoxyribonucleic acid; CIC: Capicua transcriptional repressor gene; $\mathrm{CNA}(\mathrm{s})$ : Copy number alteration(s); CNS: Central nervous system; DICER1: Dicer 1, ribonuclease III gene; DLEU1: Deleted In Lymphocytic Leukemia 1; DNAJC12: DnaJ Heat Shock Protein Family (Hsp40) Member C12 gene; dNTP(s): Deoxyribonucleotide triphosphate(s); dsDNA: Double stranded deoxyribonucleic acid; DXA: Dual-energy X-ray absorptiometry; eES: Enhanced exome sequencing; EMA: Epithelial membrane antigen; ETMR: Embryonal tumor with multilayered rosettes; EWSR1: Ewing sarcoma breakpoint region 1 gene; FISH: Fluorescence in-situ hybridization; G-tube: Gastrostomy tube; GFAP: Glial fibrillary acid protein; Gl: Gastrointestinal; GRCh37: Genome Reference Consortium Human Build 37; GRCh38: Genome Reference Consortium Human Build 38; GRCh38.p13: Genome Reference Consortium Human Build 38 patch release 13; GS: Genome sequencing; H\&E: Hematoxylin and eosin; IHC: Immunohistochemical; IGM: The Steve and Cindy Rasmussen Institute for Genomic Medicine (IGM); indel: Insertion-deletion; INI1: Integrase interactor 1; IRB: Institutional Review Board; KLHL1: Kelch-like 1 gene; LINE: Long interspersed nuclear element; LIN28: Lin-28 homolog A; LOH: Loss of heterozygosity; MRI: Magnetic resonance imaging; MYCN: N-MYC proto-oncogene; $\mathrm{NCH}$ : Nationwide Children's Hospital; Neu-N: Neuronal nuclei protein; NGS: Next generation sequencing; NOS: Not otherwise specified; nt(s): Nucleotide(s); 
NUTM1: Nuclear protein in testis family member 1 protein; PacBio: Pacific Biosciences; PBMCs: Peripheral blood mononuclear cells; PCR: Polymerase chain reaction; $\mathrm{QPCR}$ : Quantitative polymerase chain reaction; $\mathrm{RB} 1$ : Retinoblastoma protein; RB1: RB Transcriptional Corepressor 1 gene; RBP3: Retinol Binding Protein 3 gene; RNA-seq: RNA-sequencing; RT-PCR: Reverse transcriptase polymerase chain reaction; SIAH3: Siah E3 Ubiquitin Protein Ligase Family Member 3 gene; SMRT: Single Molecule Real Time sequencing; SNV(s): Single nucleotide variant(s); SV(s): Structural variation(s); TPM: Transcripts per million; TPT1:Tumor Protein, Translationally-Controlled 1 gene; t-SNE: T-Distributed Stochastic Neighbor Embedding; ZC3H13: Zinc Finger CCCH-Type Containing 13 gene.

\section{Supplementary Information}

The online version contains supplementary material available at https://doi. org/10.1186/s40478-021-01164-z.

Additional file 1 Supplementary methods and data.

\section{Acknowledgements}

We thank the patient and their family for participating in our translational research protocol. We thank the Nationwide Foundation Pediatric Innovation Fund for generously supporting sequencing, data production, and analysis. Preliminary data was presented at the American Association of Neuropathology annual meeting in Atlanta, GA in June, 2019. E.A.K. was supported by the National Institute of General Medical Sciences of the National Institutes of Health under Award Number 2T32GM068412-11A1. The content is solely the responsibility of the authors and does not necessarily represent the official views of the National Institutes of Health.

\section{Authors' contributions}

KMS and AZF both drafted the manuscript KMS, EAK, SM, AM, MEH-G, SL, KEM, DCK. PB, BK, AW, PW, VM, ERM, RKW, and CEC. contributed to the extensive molecular workup of the patient. VA, MS, SC, RAJ, AS, JLF, DSO, AID, and JRL contributed to the clinical workup of the patient. DPR contributed to the radiologic findings for the case. AZF, MS, BAO, CRP, and DRB contributed to the histopathologic, immunohistochemical, FISH, and DNA array-based methylation findings for the case. All authors read and approved the final manuscript.

\section{Funding}

We thank the Nationwide Foundation Pediatric Innovation Fund for generously supporting sequencing, data production, and analysis. E.A.K. was supported by the National Institute of General Medical Sciences of the National Institutes of Health under Award Number 2T32GM068412-11A1. The content is solely the responsibility of the authors and does not necessarily represent the official views of the National Institutes of Health.

\section{Availability of data and materials}

The datasets generated during and/or analyzed during the current study are available in dbGAP (https://www.ncbi.nlm.nih.gov/gap/) [phs001820.v1.p1].

\section{Declarations}

Ethics approval and consent to participate and consent for publication The patient was enrolled as part of an Institutional Review Board (IRB) approved study (IRB18-00662) at The Steve and Cindy Rasmussen Institute for Genomic Medicine (IGM) at Nationwide Children's Hospital (NCH). Informed consent was provided by the patient's parents for comprehensive genomic analysis and publication.

\section{Competing interests}

V.M. is a Key Opinion Leader at New England Biolabs. The remaining authors declare that they have no competing interests.

\section{Author details}

${ }^{1}$ The Steve and Cindy Rasmussen Institute for Genomic Medicine, Abigail Wexner Research Institute At Nationwide Children's Hospital, 575 Children's Crossroad, Columbus, OH 43215, USA. ${ }^{2}$ Department of Pathology,
Northwestern University Feinberg School of Medicine, Chicago, IL, USA.

${ }^{3}$ Department of Pediatrics, The Ohio State University College of Medicine, Columbus, OH, USA. ${ }^{4}$ Division of Hematology/Oncology, Department of Pediatrics, Nemours Children's Health System, Orlando, FL, USA. ${ }^{5}$ Division of Hematology/Oncology, Department of Pediatrics, Washington University School of Medicine in St. Louis, St. Louis, MO, USA. ${ }^{6}$ Division of Hematology, Oncology, and Bone Marrow Transplant, Nationwide Children's Hospital, Columbus, OH, USA. ${ }^{7}$ Department of Radiology, Nationwide Children's Hospital, Columbus, OH, USA. ${ }^{8}$ Division of Gastroenterology \& Hepatology \& Nutrition, Nationwide Children's Hospital, Columbus, OH, USA. ${ }^{9}$ Department of Pathology, New York University Langone Health, New York City, NY, USA. ${ }^{10}$ Department of Pathology, St. Jude Children's Research Hospital, Memphis, TN, USA.

${ }^{11}$ Division of Hematology and Oncology, The Ohio State University College of Medicine, Columbus, OH, USA. ${ }^{12}$ Departments of Pediatrics and Radiation Oncology, The Ohio State University College of Medicine, Columbus, OH, USA. ${ }^{13}$ Division of Neurosurgery, Nationwide Children's Hospital, Columbus, OH, USA. ${ }^{14}$ Department of Neurosurgery, The Ohio State University College of Medicine, Columbus, OH, USA. ${ }^{15}$ Department of Pathology and Laboratory Medicine, Nationwide Children's Hospital, Columbus, OH, USA. ${ }^{16}$ Department of Pathology, The Ohio State University College of Medicine, Columbus, $\mathrm{OH}$ , USA. ${ }^{17}$ Department of Biomedical Education \& Anatomy, The Ohio State University, Columbus, OH, USA.

Received: 5 February 2021 Accepted: 19 March 2021

Published online: 07 April 2021

\section{References}

1. Capper D, Jones DTW, Sill M, Hovestadt V, Schrimpf D, Sturm D et al (2018) DNA methylation-based classification of central nervous system tumours. Nature 555:469-474. https://doi.org/10.1038/nature26000

2. Davies HR, Broad KD, Onadim Z, Price EA, Zou X, Sheriff I et al (2021) Whole-genome sequencing of retinoblastoma reveals the diversity of rearrangements disrupting RB1 and uncovers a treatment-related mutational signature. Cancers (Basel) 13:754. https://doi.org/10.3390/cance rs13040754

3. Dimaras H, Corson TW, Cobrinik D, White A, Zhao J, Munier FL et al (2015) Retinoblastoma. Nat Rev Dis Prim. https://doi.org/10.1038/nrdp.2015.21

4. Dunkel IJ, Jubran RF, Gururangan S, Chantada GL, Finlay JL, Goldman S et al (2010) Trilateral retinoblastoma: potentially curable with intensive chemotherapy. Pediatr Blood Cancer 54:384-387. https://doi.org/10. 1002/pbc.22336

5. Elias WJ, Lopes MBS, Golden WL, Jane S, Gonzalez-Fernandez F (2001) Trilateral retinoblastoma variant indicative of the relevance of the retinoblastoma tumor-suppressor pathway to medulloblastomas in humans. J Neurosurg 95:871-878. https://doi.org/10.3171/jns.2001.95.5.0871

6. Finelli DA, Shurin SB, Bardenstein DS (1995) Trilateral retinoblastoma: Two variations. Am J Neuroradiol 16:166-170

7. Gupta VA, Beggs AH (2014) Kelch proteins: Emerging roles in skeletal muscle development and diseases. Skelet Muscle 4:11

8. Harbour JW (2001) Molecular basis of low-penetrance retinoblastoma. Arch Ophthalmol 119:1699-1704

9. Harbour JW, Dean DC (2000) The Rb/E2F pathway: Expanding roles and emerging paradigms. Genes Dev 14:2393-2409. https://doi.org/10.1101/ gad. 813200

10. Heller D, Vingron M (2019) SVIM: structural variant identification using mapped long reads. Bioinformatics 35:2907-2915

11. Kamihara J, Bourdeaut F, Foulkes WD, Molenaar JJ, Mosse YP et al (2017) Retinoblastoma and Neuroblastoma predisposition and surveillance. Clin Cancer Res 23:e98-e106. https://doi.org/10.1158/1078-0432.CCR-17-0652

12. Karimi S, Zuccato JA, Mamatjan Y, Mansouri S, Suppiah S et al (2019) The central nervous system tumor methylation classifier changes neuro-oncology practice for challenging brain tumor diagnoses and directly impacts patient care. Clin Epigenetics. https://doi.org/10.1186/ s13148-019-0766-2

13. Kingston JE, Plowman PN, Hungerford JL (1985) Ectopic intracranial retinoblastoma in childhood. Br J Ophthalmol 69:742-748. https://doi. org/10.1136/bjo.69.10.742 
14. Knudson AG (1971) Mutation and cancer: statistical study of retinoblastoma. Proc Natl Acad Sci U S A 68:820-823. https://doi.org/10.1073/pnas. 68.4.820

15. Kooi IE, Mol BM, Massink MPG, Ameziane N, Meijers-Heijboer H et al (2016) Somatic genomic alterations in retinoblastoma beyond RB1 are rare and limited to copy number changes. Sci Rep 6:25264. https://doi. org/10.1038/srep25264

16. Korbel JO, Campbell PJ (2013) Criteria for inference of chromothripsis in cancer genomes. Cell 152:1226-1236

17. Li H (2018) Minimap2: pairwise alignment for nucleotide sequences. Bioinformatics 34:3094-3100

18. MacDonald JR, Ziman R, Yuen RKC, Feuk L, Scherer SW (2014) The database of genomic variants: a curated collection of structural variation in the human genome. Nucleic Acids Res. https://doi.org/10.1093/nar/ gkt958

19. Mantere T, Kersten S, Hoischen A (2019) Long-read sequencing emerging in medical genetics. Front Genet 10:426

20. McEvoy J, Nagahawatte P, Finkelstein D, Richards-Yutz J, Valentine M et al (2014) RB1 gene inactivation by chromothripsis in human retinoblastoma. Oncotarget 5:438-450. https://doi.org/10.18632/oncotarget.1686

21. Price EA, Price K, Kolkiewicz K, Hack S, Reddy MA, Hungerford JL et al (2014) Spectrum of RB1 mutations identified in 403 retinoblastoma patients. J Med Genet 51:208-214. https://doi.org/10.1136/jmedg enet-2013-101821

22. Richter S, Vandezande K, Chen N, Zhang K, Sutherland J, Anderson J et al (2003) Sensitive and efficient detection of RB1 gene mutations enhances care for families with retinoblastoma. Am J Hum Genet 72:253-269. https://doi.org/10.1086/345651

23. Römer T, Temming P, Lohmann DR, Sturm D, von Deimling A, Sellhaus $B$ et al (2019) Ectopic intracranial retinoblastoma in a 3.5-month-old infant without eye involvement and without evidence of heritability. Pediatr Blood Cancer 66:1-5. https://doi.org/10.1002/pbc.27599

24. Rushlow DE, Mol BM, Kennett JY, Yee S, Pajovic S, Thériault BL et al (2013) Characterisation of retinoblastomas without RB1 mutations: Genomic, gene expression, and clinical studies. Lancet Oncol 14:327-334. https:// doi.org/10.1016/S1470-2045(13)70045-7

25. Sakamoto Y, Sereewattanawoot S, Suzuki A (2020) A new era of long-read sequencing for cancer genomics. J Hum Genet 65:3-10

26. Santagata S, Maire CL, Idbaih A, Geffers L, Correll M, Holton K et al (2009) CRX is a diagnostic marker of retinal and pineal lineage tumors. PLoS ONE. https://doi.org/10.1371/journal.pone.0007932

27. Sapède D, Cau E (2013) The Pineal Gland from development to function. In: Current topics in developmental biology. Academic Press Inc., pp $171-215$
28. Sedlazeck FJ, Rescheneder P, Smolka M, Fang H, Nattestad M, Von Haeseler A et al (2018) Accurate detection of complex structural variations using single-molecule sequencing. Nat Methods 15:461-468. https://doi. org/10.1038/s41592-018-0001-7

29. Skalet AH, Gombos DS, Gallie BL, Kim JW, Shields CL, Marr BP et al (2018) Screening children at risk for retinoblastoma: consensus report from the American association of ophthalmic oncologists and pathologists. Ophthalmology 125:453-458. https://doi.org/10.1016/j.ophtha.2017.09. 001

30. Stephens PJ, Greenman CD, Fu B, Yang F, Bignell GR, Mudie LJ et al (2011) Massive genomic rearrangement acquired in a single catastrophic event during cancer development. Cell 144:27-40. https://doi.org/10.1016/j. cell.2010.11.055

31. Torczynski E, Jacobiec FA, Johnston MC, Font RL, Madewell JA (1977) Synophthalmia and cyclopia: a histopathologic, radiographic, and organogenetic analysis. Doc Ophthalmol 44:311-378. https://doi.org/10. $1007 /$ bf00230088

32. Valverde JR, Alonso J, Palacios I, Pestaña Á (2005) RB1 gene mutation up-date, a meta-analysis based on 932 reported mutations available in a searchable database. BMC Genet 6:53. https://doi.org/10.1186/ 1471-2156-6-53

33. Wenger AM, Peluso P, Rowell WJ, Chang PC, Hall RJ, Concepcion GT et al (2019) Accurate circular consensus long-read sequencing improves variant detection and assembly of a human genome. Nat Biotechnol 37:1155-1162. https://doi.org/10.1038/s41587-019-0217-9

34. Wright KD, Qaddoumi I, Patay Z, Gajjar A, Wilson MW, Rodriguez-Galindo C (2010) Successful treatment of early detected trilateral retinoblastoma using standard infant brain tumour therapy. Pediatr Blood Cancer 55:570-572. https://doi.org/10.1002/pbc.22545

35. Yamanaka R, Hayano A, Takashima Y (2019) Trilateral retinoblastoma: a systematic review of 211 cases. Neurosurg Rev 42:39-48

36. Zhang J, Walsh MF, Wu G, Edmonson MN, Gruber TA, Easton J et al (2015) Germline mutations in predisposition genes in pediatric cancer. N Engl J Med 373:2336-2346. https://doi.org/10.1056/NEJMoa1508054

\section{Publisher's Note}

Springer Nature remains neutral with regard to jurisdictional claims in published maps and institutional affiliations.
Ready to submit your research? Choose BMC and benefit from:

- fast, convenient online submission

- thorough peer review by experienced researchers in your field

- rapid publication on acceptance

- support for research data, including large and complex data types

- gold Open Access which fosters wider collaboration and increased citations

- maximum visibility for your research: over $100 \mathrm{M}$ website views per year

At BMC, research is always in progress.

Learn more biomedcentral.com/submissions 